\title{
Dynamic growth effects during low-pressure deposition of diamond films
}

Donald R. Gilberta) and Rajiv Singh

Department of Materials Science and Engineering, University of Florida, Gainesville, Florida 32611

Roy Clarke and S. Murugkar

Department of Applied Physics, University of Michigan, Ann Arbor, Michigan 48109

(Received 9 September 1996; accepted for publication 8 February 1997)

\begin{abstract}
Diamond films were deposited in a modified electron-cyclotron-resonance plasma system operating at pressures between 1.0 and 2.0 Torr. This system provides the advantage of efficient plasma generation due to magnetic enhancement and high diffusion rates due to relatively low-pressure operation. Films were formed from preexisting seed layers providing high " nucleation', densities to promote rapid coalescence. Raman analysis of grown films showed a quality dependence on both deposition pressure and nucleation density. We speculate that the increased presence of amorphous carbon and larger film stresses is the result of grain-boundary impurity effects in the seeded films. Oxygen addition improved film quality by reducing nondiamond carbon incorporation. (C) 1997 American Institute of Physics. [S0003-6951(97)03915-6]
\end{abstract}

Diamond chemical vapor deposition (CVD) is typically accomplished in a pressure range of 20-60 Torr at substrate temperatures ranging from 750 to $950{ }^{\circ} \mathrm{C} .{ }^{1-5}$ However, deposition of diamond under lower pressure conditions (pressure less than 2 Torr) provides several advantages. In a lowpressure plasma system, the electron temperature may be much higher than the gas temperature, particularly when magnetic enhancement is applied, as in the case of electroncyclotron resonance (ECR). ${ }^{6}$ The low neutral gas temperature $\left(\sim 500{ }^{\circ} \mathrm{C}\right)$ of such a system is expected to significantly reduce contamination in the growing film. Low pressure also results in a more uniform, large area plasma with longer mean-free-path lengths and higher diffusion rates, which are critical for large area growth. Due to the plasma-enhanced nature of deposition in this system, necessary deposition temperatures are effectively reduced, allowing a greater potential range of substrate materials to be used. Deposition at lower temperature may also reduce the residual stress due to thermal expansion mismatch, depending on the substrate material. Application of ECR plasma systems to diamond CVD has been the subject of increasing interest in recent years. ${ }^{7-10}$ The typical deposition pressures for diamond films fabricated by the ECR process have been between 10 and 100 mTorr. ${ }^{9,10}$ However, deposition of high-quality films has not been readily realized under these conditions. The films formed under these conditions are typically characterized by $s p^{2}$ impurities and wide [full width at half-maximum (FWHM) 10-20 $\mathrm{cm}^{-1}$ ] Raman peak characteristics of the diamond phase. In addition, external biasing has been found necessary for deposition of the diamond phase at these pressures. $^{10}$

Experiments were conducted in a Plasma-Therm BECR-6 electron-cyclotron-resonance plasma system. The details of the system have been discussed elsewhere. ${ }^{9}$ This system uses a series of permanent rare-earth magnets to produce the necessary magnetic field of $875 \mathrm{G}$ in the plasma generation zone that matches the cyclotron frequency of the free electrons to the $2.45 \mathrm{GHz}$ microwaves. Silicon substrates were either scratched or seeded with submicron dia-

${ }^{\text {a)} E l e c t r o n i c ~ m a i l: ~ d g i l b @ m a i l . m s e . u f l . e d u ~}$ mond particles. The seeding of the surface facilitated the formation of continuous films over the relatively short growth times $(\sim 5 \mathrm{~h})$. Diamond films were deposited from gas mixtures of $1 \%$ methanol $\left(\mathrm{CH}_{3} \mathrm{OH}\right)$ by volume in hydrogen at a substrate temperature of approximately $700{ }^{\circ} \mathrm{C}$ and microwave power of $1000 \mathrm{~W}$. Samples were also deposited with additional oxygen to examine the effect on resulting film quality. Although diamond films have been formed at much lower substrate temperatures $\left(\sim 500-550{ }^{\circ} \mathrm{C}\right)$ in this system, ${ }^{11}$ this substrate temperature was chosen as a standard to analyze the effect of system pressure, which was varied from 1.00 to 1.90 Torr. The average diamond growth rates were approximately $0.2 \mu \mathrm{m} / \mathrm{h}$. The samples were analyzed by Raman spectroscopy and scanning electron microscopy (SEM).

An important aspect of these experiments was the effect of relative nucleation density on the film quality. Nucleation density was controlled by either scratching the substrate or by controlled electrophoretic seeding of the substrate. Figure 1 shows the surface morphology of a diamond-seeded silicon substrate. The surface was seeded by preparing a dispersed diamond colloid in acetone and depositing the particles on the surface using the electrophoretic method. ${ }^{12}$ The micrograph shows that the silicon surface has a submicron (aver-

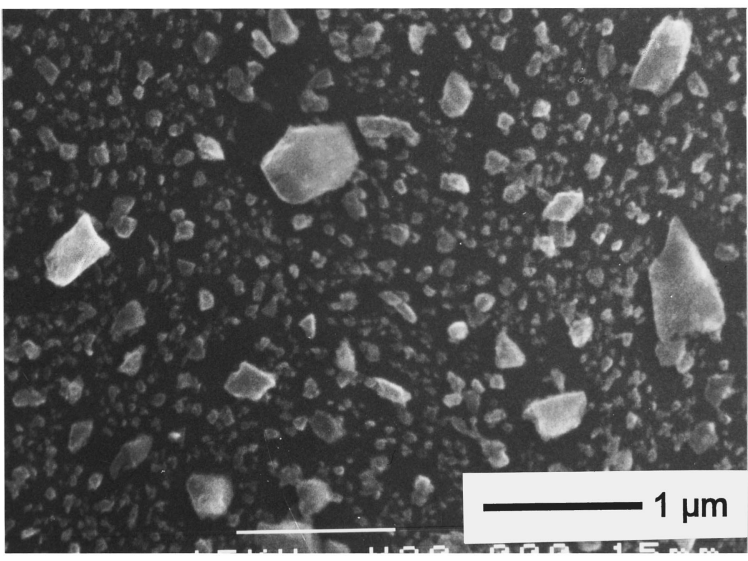

FIG. 1. SEM micrograph showing diamond seed particles on silicon substrate. 

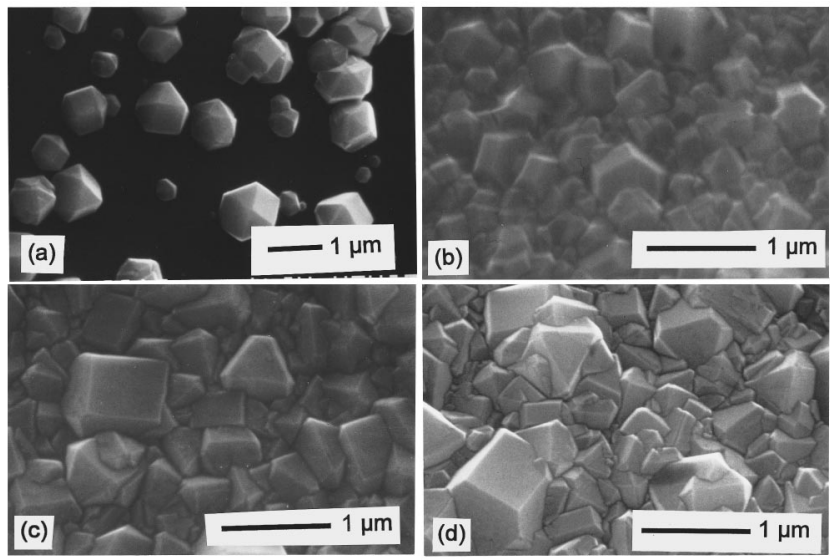

FIG. 2. SEM micrographs of resulting deposits at (a) low nucleation density and 1.50 Torr and high nucleation density at (b) 1.00, (c) 1.50, and (d) 1.90 Torr after $5 \mathrm{~h}$ at $700^{\circ} \mathrm{C}$.

age size $0.20 \mu \mathrm{m})$ diamond seed layer with a particle concentration of approximately $5 \times 10^{9} \mathrm{~cm}^{-2}$. An advantage of the electrophoretic method is the high uniformity of the dispersed diamond particles on the surface. The seed density was controlled from $10^{9}$ to $10^{10} \mathrm{~cm}^{-2}$ by controlling the deposition time and the concentration of diamond powder in the suspension. It should be noted that, under standard ECR deposition conditions, the density of nuclei obtained from scratching techniques is much lower $\left(\sim 10^{8} \mathrm{~cm}^{-2}\right)$ than obtained from the electrophoretic process.

The surface morphology of diamond films grown for $5 \mathrm{~h}$ under various chamber pressures $(1.00,1.50$, and 1.90 Torr) is shown in Fig. 2. In all these cases, the substrate temperature of $700{ }^{\circ} \mathrm{C}$ and nucleation density in the range of $10^{9} \mathrm{~cm}^{-2}$ was maintained. Figure 2 shows that faceted films were deposited under all pressures. The high nucleation density resulted in formation of small diamond grains due to impingement during crystal growth (average grain size $\sim 0.5$ $\mu \mathrm{m})$. At 1.00 Torr, smooth overall faceting is observed with small average crystallite structure. A larger average crystal structure was obtained at 1.50 Torr. The (100) facets appear smooth and there is evidence of very small scale roughness on the (111) facets. Deposition at 1.90 Torr demonstrated nearly identical results to the 1.50 Torr deposited film. Also shown in Fig. 2 is the surface morphology of a diamond film deposited for $5 \mathrm{~h}$ on a scratched silicon substrate at 1.50 Torr. In contrast to the above results, discontinuous film growth was observed that is the result of the low nucleation density. However, the Raman results (discussed in the next paragraph) suggest the quality of the deposited diamond was better than the higher nucleation density films deposited under the same conditions.

Macro-Raman spectroscopy was conducted on these samples to characterize the structural composition of the resultant films. Figure 3 shows the corresponding Raman spectra obtained from the films of Fig. 2. Spectra were normalized with respect to maxima and minima for comparison. Due to its optical transparency, Raman scattering intensity is directly affected by the thickness of the diamond film. A strong characteristic diamond peak at approximately $\sim 1334$ $\mathrm{cm}^{-1}$ was obtained for the samples grown under these conditions. The full width at half-maximum of the characteristic

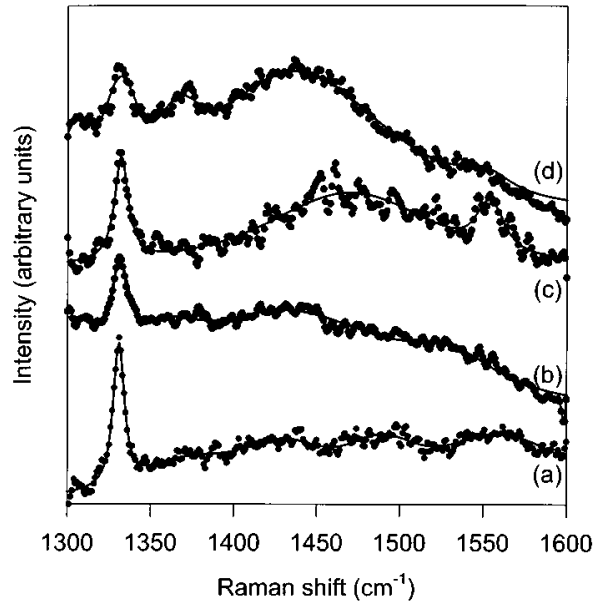

FIG. 3. Raman spectra of films shown in Fig. 2 deposited from $\mathrm{CH}_{3} \mathrm{OH} / \mathrm{H}_{2}$ gas mixture with (a) low nucleation density at 1.5 Torr and high nucleation density at (b) 1.0, (c) 1.5, and (d), 1.9 Torr.

diamond peaks varied from 8 to $10 \mathrm{~cm}^{-1}$. The broadening in the peak for high nucleation density samples can be attributed to defects, small domain size, intrinsic stress, and the presence of graphitic impurities in the film. Also, curve fitting of the spectra indicated the presence of two other broad peaks, one positioned at about $1510 \mathrm{~cm}^{-1}$ and the other about $1450 \mathrm{~cm}^{-1}$. These signals are attributed to diamondlike $\left(1510 \mathrm{~cm}^{-1}\right)$ and hydrogenated amorphous carbon $\left(1450 \mathrm{~cm}^{-1}\right)$.

The film quality measured by Raman spectroscopy was found to be strongly dependent on the initial nucleation density. For lower nucleation densities, the film quality was found to improve significantly. Figure 3(a) shows a spectrum taken from a film deposited at 1.50 Torr with a relatively low nucleation density. The diamond peak for this sample has a substantially smaller FWHM $\left(7.7 \mathrm{~cm}^{-1}\right)$ than a film deposited simultaneously with a higher nucleation density (FWHM $11.4 \mathrm{~cm}^{-1}$ ). Besides the broadening of the diamond peak on the seeded sample, the characteristic peak showed shifting to higher wave numbers (an increase of approximately $\sim 1.0$ $\mathrm{cm}^{-1}$ ). This is an indication of greater compressive stress in the film deposited from a higher nucleation density, which in part may account for the observed peak broadening. ${ }^{13}$ Also, the nondiamond impurities were substantially reduced in the film deposited with lower nucleation. We speculate that this is an indication of grain boundaries influencing the incorporation of nondiamond carbon as the film grows.

The addition of oxygen during deposition was found to improve film quality for both low and high nucleation density films. Figure 4 shows the Raman spectra for continuous films deposited for $7.5 \mathrm{~h}$ simultaneously on both seeded and scratched silicon substrates using a gas mixture of $1.2 \%$ $\mathrm{CH}_{3} \mathrm{OH}+0.3 \% \mathrm{O}_{2}+98.5 \% \mathrm{H}_{2}$. The characteristic diamond peak was strong for both specimens and secondary nondiamond peaks were not readily identifiable, indicating a significantly improved microstructure. The film grown from preexisting seeds again showed a slightly broader diamond peak shifted slightly higher from the standard position than the film deposited on the scratched substrate. SEM showed the film grown on scratched silicon was made up of many 


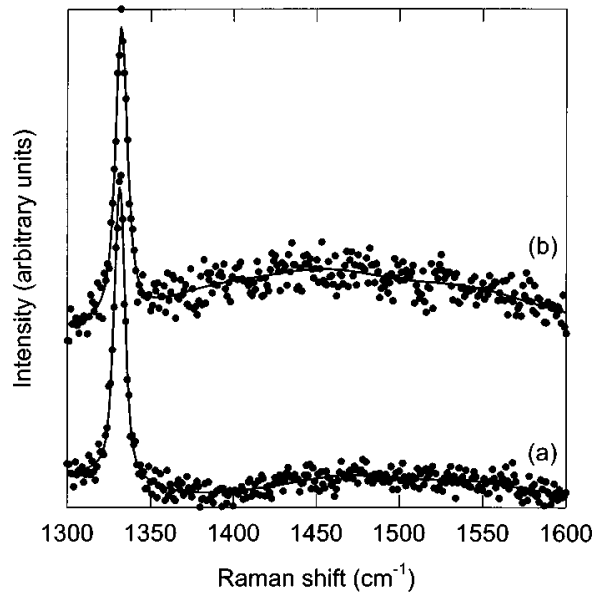

FIG. 4. Raman spectra of films deposited simultaneously with (a) high and (b) low nucleation densities from $\mathrm{CH}_{3} \mathrm{OH} / \mathrm{O}_{2} / \mathrm{H}_{2}$ source gas mixture.

regular crystallites with a relatively low incidence of twinning. The film grown from preexisting seeds has a more densely packed structure with a greater degree of crystallite impingement and twinning and smaller average crystallite size.

In conclusion, we have deposited good quality diamond thin films at low pressures ( $<2$ Torr) using an ECR plasmaenhanced CVD system. The relative quality of the diamond was found to be dependent on the initial nucleation density and, presumably, resulting grain-boundary fraction. Addition of oxygen to the source gas greatly reduced nondiamond impurities in the deposited films.

The authors would like to acknowledge the valuable assistance of Dr. W. Brock Alexander in interpreting our data. Part of this research is sponsored by the National Science Foundation, through the Engineering Research Center on Particle Science and Technology Grant No. EEC 94-02989.

${ }^{1}$ P. K. Bachmann, D. Leers, and H. Lydtin, Diam. Relat. Mater. 1, 1 (1991).

${ }^{2}$ N. Setaka, Carbon 28, 764 (1990).

${ }^{3}$ K. McNamara and K. K. Gleason, J. Electrochem. Soc. 140, L22 (1993).

${ }^{4}$ K. Kobashi, K. Nishimura, K. Miyata, K. Kumagai, and A. Nakave, J. Mater. Res. 5, 2469 (1990).

${ }^{5}$ Y. Liou, A. Inspektor, R. Weimer, D. Knight, and R. Messier, J. Mater. Res. 5, 2305 (1990)

${ }^{6}$ Jes Asmussen, J. Vac. Sci. Technol. A 7, 883 (1989).

${ }^{7}$ A. Hiraki, H. Kawarada, J. Wei, J. S. Ma, and J. Suzuki, Proc. SPIE 1325, 73 (1990).

${ }^{8}$ Y. H. Shing, F. S. Pool, and D. H. Rich, Thin Solid Films 212, 150 (1992).

${ }^{9}$ R. K. Singh, D. Gilbert, R. Tellshow, P. H. Holloway, R. Ochoa, J. H. Simmons, and R. Koba, Appl. Phys. Lett. 61, 2863 (1992).

${ }^{10}$ C. R. Eddy, D. L. Youchison, and B. D. Sartwell, Diam. Relat. Mater. 3, 105 (1994).

${ }^{11}$ R. K. Singh, D. R. Gilbert, J. Fitz-Gerald, S. Harkness, and D. G. Lee, Science 272, 396 (1996).

${ }^{12}$ R. K. Singh and D. G. Lee, Mater. Res. Soc. Symp. Proc. (in press).

${ }^{13}$ L. Bergman and R. J. Nemanich, J. Appl. Phys. 78, 6709 (1995). 